\title{
Guided-wave based structural health monitoring of built-up composite structures using spectral finite element method
}

\author{
Ajith V. ${ }^{a}$ and Goapalakrishnan. $\mathrm{S}^{a}$ \\ ${ }^{a}$ Department of Aerospace Engineering, Indian Institute of Science, Bangalore, \\ India-560012;
}

\begin{abstract}
The paper discusses basically a wave propagation based method for identifying the damage due to skin-stiffener debonding in a stiffened structure. First, a spectral finite element model (SFEM) is developed for modeling wave propagation in general built-up structures, using the concept of assembling 2D spectral plate elements and the model is then used in modeling wave propagation in a skin-stiffener type structure. The damage force indicator (DFI) technique, which is derived from the dynamic stiffness matrix of the healthy stiffened structure (obtained from the SFEM model) along with the nodal displacements of the debonded stiffened structure (obtained from 2D finite element model), is used to identify the damage due to the presence of debond in a stiffened structure.
\end{abstract}

Keywords: Stiffened structure, wave propagation, SFEM, skin-stiffener debond, damage model

\section{INTRODUCTION}

The stiffened composite structures are the building block of the wing sections of an aircraft. The structural health monitoring of these composite structures is a major challenge, faced by the aircraft industry. In case of composite structures, guided waves offer a great potential for health monitoring applications since their propagation mechanism interact strongly even with small damage. Further, these waves can be excited and sensed by piezoelectric elements that can be permanently attached onto a structure offering online monitoring capability. The structural health monitoring of stiffened plate structure and wing box structure, using guided wave technique can be found respectively in, Rathod and Mahapatra $2010^{1}$ and Grondal et.al. ${ }^{2}$. However, due to the difficulty in setting up the experiment, there is always a severe demand for a numerical model of these built-up structures.

There are several numerical methods found in the archival of literature for the vibration analysis of stiffened plate and among them the finite element $(\mathrm{FE})$ based method has been found to be accurate with less complexity to model stiffened plates analyzed the wave propagation in ribstiffened plate by considering the built-up plate as an equivalent orthotropic plate and using a semianalytical finite element technique ${ }^{3-} .9$ This work is limited to analyze the freely propagating waves. With the application of a load, the criteria for choice of an equivalent structure are more extensive, as the corresponding impedances must also be matched in addition to matching the wavenumbers for propagating waves. Further, these FE based models are computationally expensive, when used for the wave propagation analysis. In a conventional $\mathrm{FE}$ analysis, the maximum possible size of the finite element depends on the wavelength of the propagating wave (Ref. 11 and Ref. 12). Hence, for high frequency wave propagation analysis, a very dense finite element mesh is inevitable to accurately

Further author information: (Send correspondence to Ajith V)

Ajith V.: E-mail: ajith@aero.iisc.ernet.in, Telephone: +91-080 22932438

Goapalkrishnan S..: E-mail: krishnan@aero.iisc.ernet.in, Telephone: +91-080 22933019 
simulate the wave propagation including the effects of wave scattering at structural discontinuities. In FE, the modeling involves a crude error-bound approximation due to the numerical stability limit in computation. Schulte et al. ${ }^{13}$ used the Gauss-Lobatto-Legendre (GLL) spectral element discretization based upon quadrangular elements for the wave propagation analysis of isotropic and anisotropic shell-structures and stiffened panels. The model is computationally efficient and when compared to the FE, only five to six nodes (depending on the degree of the interpolation polynomial) per shortest wavelength of the excited frequency range are necessary to capture the structural behavior with the same accuracy as 15-30 nodes, which are needed using lower order FE. However, in the present work, we use the spectral plate element, which is found in Ref. 10, as a building block to model stiffened plate and the box-type structures. In SFEM, to model a healthy plate element, however long the plate may be, the model needs only two nodes, which reduces the system size drastically. Using this 2D plate element, any 3D structures can be modeled easily using the concept of assembly of elements and transformation from local co-ordinate to global co-ordinate. Hence, in this work, the stiffness matrix is transformed from local co-ordinate to global co-ordinate, using a transformation matrix (Ref. 11) to build the model for 3D built-up structure from a 2D spectral plate element model.

The paper is organized as follows. In the next section, description of the development of plate element, using SFEM is given, which is followed by the brief description of the method of modeling built-up structures. The SFEM model thus developed for built-up structures is then used to perform the high frequency wave propagation analysis of a healthy skin-stiffener structure. The responses obtained using the SFEM model is first validated with 2D FE results and the model is then used to detect the damage due to debonding in a skin-stiffener structure using the method of DFI.

\section{SPECTRAL ELEMENT FORMULATION OF COMPOSITE PLATES}

Spectral composite plate formulation is reported in [Gopalakrishnan et.al.2008, and Abir and Gopalakrishnan 2006]. Here, the formulation is briefly discussed for completeness. The governing equations for a plate for FLPT can be obtained by applying Hamilton's principle and they can be written as (Ref. 10),

$$
\begin{gathered}
\frac{\partial N_{x x}}{\partial x}+\frac{\partial N_{x y}}{\partial y}=I_{0} \frac{\partial^{2} u}{\partial t^{2}}-I_{1} \frac{\partial^{2} \phi}{\partial t^{2}} \\
\frac{\partial N_{x y}}{\partial x}+\frac{\partial N_{y y}}{\partial y}=I_{0} \frac{\partial^{2} v}{\partial t^{2}}-I_{1} \frac{\partial^{2} \psi}{\partial t^{2}} \\
\frac{\partial V_{x}}{\partial x}+\frac{\partial V_{y}}{\partial y}=I_{0} \frac{\partial^{2} w}{\partial t^{2}} \\
\frac{\partial M_{x x}}{\partial x}+\frac{\partial M_{x y}}{\partial y}=I_{2} \frac{\partial^{2} \phi}{\partial t^{2}}-I_{1} \frac{\partial^{2} u}{\partial t^{2}} \\
\frac{\partial M_{x y}}{\partial x}+\frac{\partial M_{y y}}{\partial y}=I_{2} \frac{\partial^{2} \psi}{\partial t^{2}}-I_{1} \frac{\partial^{2} v}{\partial t^{2}}
\end{gathered}
$$

where the mass moments are defined as,

$$
\left.\left[I_{0}, I_{1}, I_{2}\right]=\right] \int_{A} \rho\left[1, Z, Z^{2}\right] d A .
$$

The associated boundary conditions are

$$
\bar{N}_{x x}=N_{x x} n_{x}+N_{x y} n_{y}
$$




$$
\begin{gathered}
\bar{N}_{y y}=N_{x y} n_{x}+N_{y y} n_{y} \\
\bar{V}_{x}=V_{x} n_{x}+V_{y} n_{y} \\
\bar{M}_{x x}=-M_{x x} n_{x}-M_{x y} n_{y} \\
\bar{M}_{y y}=-M_{x y} n_{x}-M_{y y} n_{y}
\end{gathered}
$$

where $\bar{N}_{x x}$ and $\bar{N}_{y y}$ applied normal force in the X and Y directions, $\bar{M}_{x x}$ and $\bar{M}_{y y}$ are the applied moments about $\mathrm{Y}$ and $\mathrm{X}$ directions and $\bar{V}_{x}$ is the applied shear force in the $\mathrm{Z}$ direction (Fig. 1). The spectral element formulation (Ref. 10) begins by assuming harmonic waves in the time and space (Fourier series is employed in Y direction in which the model is unbounded), i.e.

$$
\left\{\begin{array}{l}
u(x, y, t) \\
v(x, y, t) \\
w(x, y, t) \\
\phi(x, y, t) \\
\psi(x, y, t)
\end{array}\right\}=\sum_{n=1}^{N} \sum_{m=1}^{M}\left\{\begin{array}{l}
\hat{u}(x) \\
\hat{v}(x) \\
\hat{w}(x) \\
\hat{\phi}(x) \\
\hat{\psi}(x)
\end{array}\right\}\left\{\begin{array}{l}
\cos \left(\eta_{m} y\right) \\
\sin \left(\eta_{m} y\right)
\end{array}\right\} e^{-j \omega_{n} t}
$$

where $u, v$, and $w$ are the displacement components of the reference plane, in the $\mathrm{X}, \mathrm{Y}$, and $\mathrm{Z}$ direction, respectively, and $\hat{u}, \hat{v}$ and $\hat{w}$ are its transform in Fourier domain. Similarly $\phi$ and $\psi$ are the rotations of the reference plane about $\mathrm{Y}$ and $\mathrm{X}$ axis, respectively and $\hat{\phi}$ and $\hat{\psi}$ are its transform in Fourier domain. Here, $\omega_{n}$ is the discrete angular frequency and $\eta_{m}$ is the discrete horizontal wavenumber $\left(\eta_{m}=2 \pi(\mathrm{m}-1) / \mathrm{Y}, \mathrm{Y}\right.$ is the length in $Y$ direction). Substituting Equation 12 in Equations 1-5 a polynomial eigen value problem is posed to find $v$ and $k$, such that

$$
\psi(k) v=\left(k^{2} A_{2}+k A_{1}+A_{0}\right) v=0
$$

where $k$ is the eigen value and $v$ is the corresponding right eigen vector and $A i$ is $5 \times 5$ matrix, which can be written in terms of the material properties. Wavenumber and the wave amplitudes are computed by a method based on companion matrix and the singular value decomposition(SVD). The force vector is evaluated at the nodes (substituting $n_{x}= \pm 1$ ), to obtain the nodal force vectors. Final equation, which shows the relation between the nodal force and nodal displacement vector at frequency $\omega_{n}$ and wavenumber $\eta_{m}$ can be written as,

$$
\{\hat{f}\}_{n, m}=\{K\}_{n, m}\{\hat{u}\}_{n, m}
$$

where $\{K\}_{n, m}$, is the dynamic stiffness matrix at frequency $\omega_{n}$ and wavenumber $\eta_{m}$ of order $10 \times$ $10,\{\hat{u}\}_{n, m}$ and $\{\hat{f}\}_{n, m}$ are nodal force and displacement vectors, respectively. Further details of the modeling has been explained in the reference (Ref. 10).

\section{MODELING OF STIFFENED STRUCTURE BY ASSEMBLING 2D PLATE ELEMENTS}

The formulated spectral plate element can also be employed for modeling built-up structures such as skin-stiffener type structures (Fig. 2), which are essential as far as aircraft structures are concerned. The stiffened structure can be modeled as an assemblage of number of plate elements. However, for analyzing three dimensional structures, it is necessary to transform the stiffness matrix from the local element coordinate to the global coordinate. The method of assembling a plate with stiffener is shown in Fig. 3. Here, skin is modeled with a plate element (1-2, Fig. 3) and its stiffness matrix can be obtained in the global coordinates (local coordinates coincides with global coordinates, XYZ as in Fig. 3) as given in Ref. 10. Stiffener is at an angle $90^{\circ}$ (anticlockwise) to the skin element 1-2 and is modeled using a plate element 2-3 (Fig. 3). The transformed coordinate system $\left(\mathrm{X}_{1} \mathrm{Y}_{1} \mathrm{Z}_{1}\right.$, 
Fig. 3) for the stiffener element formulation is obtained by rotating the global coordinate system (XYZ, Fig. 3), by $90^{0}$ anticlockwise. Hence, before assembling the the elements $1-2$ and 2-3, the stiffness matrix of the element 2-3, which is obtained in the local coordinate system $\left(\mathrm{X}_{1} \mathrm{Y}_{1} \mathrm{Z}_{1}\right.$, Fig. 3 ) is transformed in to global (XYZ, Fig. 3) coordinate system using a transformation matrix $\mathbf{T}$ of order $10 \times 10$ given by

$\mathbf{T}=\left[\begin{array}{ll}Q & 0 \\ 0 & Q\end{array}\right], \quad Q=\left[\begin{array}{ccccc}\cos \theta & 0 & \sin \theta & 0 & 0 \\ 0 & 1 & 0 & 0 & 0 \\ -\sin \theta & 0 & \cos \theta & 0 & 0 \\ 0 & 0 & 0 & 1 & 0 \\ 0 & 0 & 0 & 0 & 1\end{array}\right], \quad\left[\mathrm{K}_{g}\right]_{n, m}=\left[\mathrm{T}^{T}\right]\left[\mathrm{K}_{l}\right]_{n, m}[\mathrm{~T}]$

where $\left[\mathrm{K}_{l}\right]_{n, m}$ is the element stiffness matrix in the local coordinate system $\left(\mathrm{X}_{1} \mathrm{Y}_{1} \mathrm{Z}_{1}\right.$, Fig. 3) and $\left[\mathrm{K}_{g}\right]_{n, m}$ is the transformed stiffness matrix in global coordinates (XYZ, Fig. 3) at each $\mathrm{n}$ and $\mathrm{m}\left(\omega_{n}\right.$ and $\left.\eta_{m}\right)$ and $\theta$ is the rotation of the plate with respect to $\mathrm{Y}$ axis $\left(90^{\circ}, \mathrm{Fig}\right.$. 3). In the next step, the stiffness matrix of element 1-2 and 2-3 can be assembled as follows

$$
\begin{aligned}
& \left(K_{g n, m}(i p t, i p t)_{\text {plate-plate coupled }}=\left(K_{g}(i p t, i p t)_{n, m}\right)_{\text {plate }, 1-2}+\left(K_{g}\left(\text { ipt }_{1}, \text { ipt }_{1}\right)_{n, m}\right)_{\text {plate }, 2-3},\right) . \\
& \left(i p t=[6,7,8,9,10], \text { ipt }_{1}=[1,2,3,4,5]\right)
\end{aligned}
$$

$$
\begin{array}{r}
K_{g} n, m(i p t, i p t)_{\text {plate-plate coupled }}=\left(K_{g}(\text { ipt }, i p t)_{n, m}\right)_{\text {plate }, 1-2} \quad \text { ipt }=[1,2,3,4,5] \\
K_{g n, m}(i p t, i p t)_{\text {plate-plate coupled }}=\left(K_{g}(i, j)_{n, m}\right)_{\text {plate }, 2-3}, \quad i, j=6,7, \ldots 10, \text { ipt }=[11,12,13,14,15]
\end{array}
$$

The stiffened structure shown in Fig. 3 is modeled by assembling spectral plate elements, 1-2, 2-3, and $2-4$ and the global stiffness matrix is of the order $20 \times 20$.

\section{RESULTS AND DISCUSSIONS}

In this section, the SFEM model, based on the spectral plate element assembly (section 3), is used for obtaining the wave responses of a composite skin-stiffener structure (Figure 2). Here, both the skin and stiffener of stiffened structure are considered as laminated composite plates and hence the whole structure can be modeled by the concept of assembling spectral plate elements, as explained in section 3. The material used for the constituent laminated composite plates, is a GFRP composite, which has the following material properties: $\mathrm{E}_{1}=144.48 \mathrm{Gpa}, \mathrm{E}_{2}=\mathrm{E}_{3}=9.63 \mathrm{Gpa}, \mathrm{G}_{23}=\mathrm{G}_{13}=$ $\mathrm{G}_{12}=4.128 \mathrm{GPa}, \nu_{23}=0.3, \nu_{13}=\nu_{12}=0.02$ and $\rho_{12}=1389 \mathrm{~kg} / \mathrm{m}^{3}$. In all the study, composite laminate considered consist of 8 layers and the thickness of each layer is assumed as $1 \mathrm{~mm}$, unless specified otherwise. First, the model is validated by comparing the responses obtained from the SFEM model with that of the responses obtained using the 2D FE analysis and then applied to perform the wave propagation analysis of multiple stiffened structure. The model is then used for detecting the damage due to debonding in a ski-stiffener structure. Transverse velocity responses obtained from the structures are mainly considered in the present study. In all the study, the responses are measured at the same point, where we apply the load.

\subsection{Validation of SFEM model}

The SFEM model developed is first validated by comparing the transverse velocity responses of a stiffened structure obtained using the model with that of the responses obtained from $2 \mathrm{D}$ FE model. In SFEM, to model a skin stiffener structure with one stiffener (Fig. 2), three spectral plate element are required, which results in a system matrix of order 20. Skin-stiffener structure used for the study is $0.8 \mathrm{~m}$ in $\mathrm{X}$-direction, $1.2 \mathrm{~m}$ in $\mathrm{Y}$ direction and a stiffener of $0.5 \mathrm{~m}$ height is 
attached at $0.6 \mathrm{~m}$ away (in X-direction) from node 1 (Fig. 2). In SFEM, load is transformed in frequency domain by the FFT, where 8192 (N in Equation 12) sampling points are used. For spatial variation, 30 Fourier series coefficients ( $\mathrm{M}$ in Equation 12)) are considered. In FE analysis, structure is modeled using 4- noded plate elements and to model the symmetric part of the skin-stiffener structure, having geometric parameters as mentioned above, the analysis requires approximately 10000 elements. While solving via FE analysis, Newmark's time integration method is adopted with a time increment of $1 \mu \mathrm{s}$. In Fig. 4, the real part of the wavenumber (which propagates, Ref. 11) is plotted against frequency $\left(\eta_{m}=50\right)$ for the GFRP plate considered for the study, in order to show the different propagating wave modes. Wavenumber contains both symmetric and anti-symmetric modes. The symmetric modes are also called longitudinal modes because the average displacement over the thickness of the plate or layer is in the longitudinal direction. The anti-symmetric modes are observed to exhibit average displacement in the transverse direction and these modes are also called flexural modes. In this paper basically we also study the coupling between the symmetric longitudinal (axial) mode $u\left(\mathrm{~S}_{0}\right.$ mode) and the anti-symmetric flexural mode $w\left(\mathrm{~A}_{0}\right.$ mode). In each case, a transverse load is applied at a point and the response is measured at the same point. The structure shown in Fig. 2 is fixed at node 4.

The transverse velocity response at node 1 (Fig. 2) of the skin-stiffener structure obtained, using both SFEM and regular FE method, is shown in Fig. 5. In the velocity response, the wave form at $100 \mu \mathrm{s}$ is the incident pulse. In Fig. 5, reflection ( $\mathrm{A}_{0}$ mode) from the junction of skin and stiffener (node 2, Fig. 2) starts at $0.62 \mathrm{~ms}$ and the reflection ( $\mathrm{S}_{0}$ mode) from the top free end of stiffener (node 3, Fig. 2), which is present due to the flexural-axial coupling, starts at 0.72 ms (marked in circle, Figure 5). In wave propagation analysis, the time gap between the incident pulse and the time for first reflection is a measure of the group speeds. Hence, if we know the distance traveled by the wave and the time gap between the incident pulse and reflected pulse, we can obtain the speed of the wave. Here, in the present study, the symmetric axial mode travels (at $10000 \mathrm{~m} / \mathrm{s}$ ) five times faster than the antisymmetric flexural mode. In the plot, an excellent match can be observed between the SFEM and regular FE response, which establishes the accuracy of the SFEM model, developed for the stiffened structures. The wave propagation analysis of the stiffened structures, using FE analysis, needs large system size and consequently large computational time, when compared to the performance of the SFEM model. Further, we can see that the effect of flexural-axial coupling due to the presence of stiffeners, are well captured by the SFEM model.

\subsection{Wave propagation in stiffened structures with stiffeners of rectangular cross section}

In this section, we conduct a parametric study in a stiffened structure (Fig. 2). Here, skin and the stiffener are modeled as spectral plate element and these three plate elements are assembled, as mentioned in section 3. The composite plates considered in this study consists of 8 layers and the material properties of each plate is same as the material properties, which we used in the validation section (section 4.1). In the skin (1-2 in Fig. 2), each layer is $1 \mathrm{~mm}$ thick and the lay-up sequence is symmetric $[0]_{8}$. In all the case, the load is applied in the transverse direction and the transverse responses are plotted. Skin is fixed at node 4 (Fig. 2). Load is applied at the free end of the structure (node 1, Fig. 2) and the responses are measured at the same point. Length of the skin structure in $\mathrm{X}$ and $\mathrm{Y}$ direction and the stiffener height are given inside the bracket while explaining each results. In all the study, the length of the structure in Y direction is taken larger compared to that of its length in $\mathrm{X}$ direction in order to avoid the effect of boundary reflections from the free end.

In wave propagation analysis, the real wavenumbers will only propagate (Ref. 11). Figure 4 shows that with in the frequency range, $20 \mathrm{kHz}-100 \mathrm{kHz}, u, v$ and $w$ modes are the only propagating wave modes (real part of wavenumbers). Here, we are interested in the study of flexural-axial 
coupling present in the wave responses of the stiffened structures. Hence, in the rest of the study, we choose either a broad band pulse of band width, $70 \mathrm{kHz}$ or a tone-burst pulse, modulated at 30 $\mathrm{kHz}$ as the impact load, in order to avoid the presence of shear modes $(\phi, \psi)$.

In the first case study, the effect of the height of the stiffener, on the transverse response is studied and the results are plotted in Fig. 6(a) and Fig. 6(b) (X=1 m, Y=2 m and stiffener height varied from $0.5 \mathrm{~m}$ to $1.5 \mathrm{~m}$ ). Even though the applied load is in the transverse direction, due to the flexural-axial coupling, $\mathrm{S}_{0}$ mode is generated in the stiffener and the trace of this mode can also be seen in the transverse velocity response of the skin-stiffener structure. The reflections (marked in circles, in Fig. 6(a)) from the stiffener top (node 3, in Fig. 2) starts at $0.7 \mathrm{~ms}$, in a $0.5 \mathrm{~m}$ high stiffener, where as in a $1.5 \mathrm{~m}$ high stiffener, the reflection will only start at $0.85 \mathrm{~ms}$. The change in the time of arrival of first reflection from the stiffener with the change in the height of the stiffener can also be noticed in Fig. 6 (b) (change from $0.7 \mathrm{~ms}$ to 0.85 ), using the tone-burst signal.

In the next study, number of stiffeners in a skin-stiffener structure is increased from one to two (Fig. 7(a)) and the responses are obtained and are shown in Fig. 8. Skin is $2 \mathrm{~m}$ in $\mathrm{X}$ direction, 4 $\mathrm{m}$ in $\mathrm{Y}$ direction. In the case of single stiffener structure, stiffener is attached at $1 \mathrm{~m}$ away (in $\mathrm{X}$ direction, stiffener 2 in Fig. 7(a)) from the application of the load. In a double stiffener structure, in addition to the previous case, one more stiffener is attached at a point, $0.5 \mathrm{~m}$ from the point of application of the load (stiffener 1 in Fig. $7(\mathrm{a})$ ). With the addition of a stiffener, additional reflections (both $\mathrm{S}_{0}$ and $\mathrm{A}_{0}$ ) are present (starts at $0.5 \mathrm{~ms}$, in Fig. 8(a) and $0.55 \mathrm{~ms}$, in Fig. 8(b)) in the wave responses. Hence, we can conclude that the SFEM model captures the effect of multiple stiffeners, quite efficiently.

\subsection{Identification of debonding in stiffened structure using a damage force indicator}

The concept of damage force indicator (Ref. 11), which is being derived from the dynamic stiffness matrix of the healthy structure along with the nodal displacements of the debonded stiffened structure, can be used for identifying the region of debond. Here, the damage can be located within the region of the sensing points. In SFEM, a healthy plate of any length, in a structure can be modeled using a single spectral plate element. Hence, for the damage force calculation, compared to the conventional FEM, the SFEM model requires only very less number of sensor points. Here, the dynamic stiffness matrix of the healthy stiffened structure obtained from the SFEM model and the nodal displacements obtained from the 2D FE analysis of the stiffened structure with debond, which is the surrogate experimental measurement, are used to compute the damage force indicators. The damage force indicator can be defined as,

$$
\Delta \hat{f}=\hat{K}_{h} \hat{u}_{d}-\hat{f}_{d}
$$

where the subscript $h$ stands for the healthy structure (stiffened structure with no debond) and the subscript $d$ stands for the structure with debond. $\hat{K}_{h}\left(\omega_{n}, \eta_{m}\right)$ is the global dynamic stiffness matrix at each FFT sampling frequency $\omega_{n}$, and wave number $\eta_{m}$ which can be obtained using the spectral finite model discussed in section 3 and the velocity response from the debonded structure, $\hat{u}_{d}$ can be obtained by performing the $2 \mathrm{D}$ FE analysis, similar to the analysis, which we performed in section 4.1. In the 2D FE analysis, debond in the skin-stiffener junction is modeled by assuming a very low stiffness properties for the elements representing the debonded area (marked in Fig. $7(\mathrm{~b})$ ), keeping all the other parameters same as we used in section 4.1. However, in this work, the term $\hat{f}_{d}$ is neglected (Equation 18) as in Ref. 11. The values of vector $\Delta \hat{f}$, associated with the degrees of freedom (dofs (element 2-3, Fig. 3) will be non-zero. The damage force indicator vector $d$ of length $\mathrm{m}$ ( $\mathrm{m}$ is the total number of dofs in the model) can be written as

$$
d_{i}=\sum_{\omega_{n}} \sum_{\eta_{m}}\left|R_{i i}\right|, \quad i \in[1, m] \quad n=1, \ldots, N m=1, \ldots, M
$$


where

$$
\hat{R}(\omega n, \eta m)=\Delta \hat{f} \Delta \hat{f}^{*}
$$

$\hat{R}\left(\omega_{n}, \eta_{m}\right)$ is an $\mathrm{m} \times \mathrm{m}$ square matrix and $\Delta \hat{f}^{*}$ is the transpose of the complex conjugate of the $\Delta \hat{f}$. In this method, if the $i$ th element includes the damaged area, there will be non-zero diagonal entries in the $\hat{R}(\omega n, \eta m)$, corresponding to the dofs associated with the $i$ th element and the rest of the entries will be zero. Magnitude of these non-zero diagonal entries will depend on the applied load, and the configuration of the debonded region. Damage force indicator vector $d$ is thus the sum of absolute values of the diagonal entries in $\hat{R}(\omega n, \eta m)$ over all frequency steps $n=1, \ldots, N$ ( $N=$ Nyquist frequency in FFT) and for each horizontal wavenumber $\left(\eta_{m}, \mathrm{~m}=1, \ldots, \mathrm{M}\right)$.

The nodal displacements obtained from the 2D FE analysis (at nodes 1, 2, 3 and 4, Fig. 2) is first converted to frequency domain before we actually multiply it with the dynamic stiffness matrix of the healthy stiffened structure (used in section 4.1), obtained from the SFEM model. The value of damage force $(d)$ is normalized with respect to $\operatorname{Max}(d)$, separately for axial and transverse dofs. A broad band load (used in section 4) is used for the study. The material and geometric properties are same as that we used in section 4.1. The plots of the normalized damage force indicator, for a stiffened structure with a debond (length in $\mathrm{Y}$ direction $=60 \mathrm{~cm}$ and the length in $\mathrm{z}$ direction $=2$ $\mathrm{cm}$ ), is shown in Fig. 9. In Fig. 9, the normalized damage force indicators for axial and transverse dofs are shown and we can see that the dofs (dofs-4, 9 and 14 (axial, Fig. 9(a)) and dofs-6,11 and 16 (transverse, Fig. 9(b))) corresponding to the debonded region (marked in Fig. 7(b)) show peaks, which indicate the presence of debond in the structure. However, the peaks of dofs corresponding to node 4 (Fig. 3) in the skin (dof 14 (axial, Fig. 9(a)) and dof 16 (transverse, Fig. 9(b))), are very small compared to the peaks of the dofs corresponding to the stiffener (dofs 4 and 9 (axial, Fig. 9(a)) and dofs 6 and 11 (transverse, Fig. 9(b))). The result shows that the method of damage force indicator is effective in locating the region of debond in a composite stiffened structure.

\section{CONCLUSION}

A model is developed to study the wave propagation in stiffened structures, using the concept of assembling the 2D plate elements, which are spectrally formulated. The method of assembling the plate elements to model a stiffened structure is very simple and straight forward. The SFEM model shows excellent match with the $2 \mathrm{D}$ FE results. Further, the model requires only small system size and consequently less computational time, compared to 2D FE analysis while solving high frequency wave propagation problems of stiffened composite structures. The effect of flexural-axial coupling on the wave responses are well captured using the SFEM model. The model is capable of modeling a skin with multiple stiffeners. The method of damage force indicator applied to the present SFEM model is found to be effective in locating the region of debond, in a stiffened structure.

\section{REFERENCES}

1. Rathod, V.T. and Mahapatra, D.R., "Lamb wave based monitoring of plate stiffener debonding using using a circular array of piezo electric sensors", Int. J. on Smart Sensing and Intelligent Systems, 3(1), 27-44 (2010).

2. Grondal, S., Assaad, J., delebarre, C., Moulin, E., "Health monitoring of a composite wingbox structure", Ultrasonics, 42, 819-824 (2004).

3. Mukhejee A. and Mukhopadhyay M., "Finite element free vibration of eccentrically stiffened plates", Comput. Struct., 30(6), 1303-1317 (1988). 
4. Palani, G.S., Iyer, N.R., Appa Rao, T.V.S.R, " An effiicient finite element model for static and dynamic analysis of eccentrically stiffened plates/shells", Comput. and Struct., 43(4), 651-661 (1992).

5. Dong-Min Lee and In Lee, "Vibration analysis of anisotropic plates with eccentric stiffeners", Comput. Struct., 57(1), 99-105 (1995) .

6. Kolli, M. and Chandrashekhara, K., "Finite element analysis of stiffened laminated plates under transverse loading", Compos. Sci. Technol., 56, 1355-1361 (1996).

7. Edward, A.S. and Samer, A.T., "A finite element model for the analysis of stiffened laminated plates", Comput. Struct., 75(4) 369-383 (2000).

8. Gangadhara, P.B, "Linear static analysis of hat-stiffened laminated shells using finite elements", Finite elem. anal. des., 39, 1125-1138 (2003).

9. Tran Ich Thinh and Ngo Nhu Khoa, "Free vibration analysis of stiffened laminated plates using a new stiffened element", Technische Mechanik, 28(3-4), 227-236 (2008).

10. Chakraborty, A. and Gopalakrishnan, S., "A Spectral finite element model for wave propagation analysis in laminated composite plate", J. Vib. Acoust., 128, 477-488 (2006).

11. S. Gopalakrishnan, A. Chakraborty, D.R. Mahapatra, Spectral Finite Element Method, Springer-Verlag, New York, 2008.

12. Horr, A.M. and Safi, M., "Full dynamic analysis of offshore platform structures using exact Timoshenko pipe element", Transactions of the ASME, 125(3), 168-175 (2003).

13. Schulte, R.T., Fritzen, C.P., Moll, J., "Spectral element modelling of wave propagation in isotropic and anisotropic shell-structures including different types of damage", IOP Conf. Series: Materials Science and Engineering, 10, 012065 (2010).

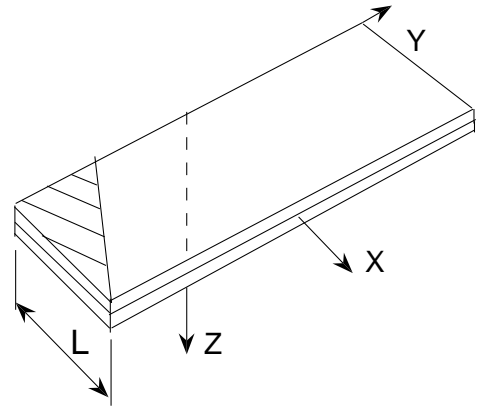

(a)

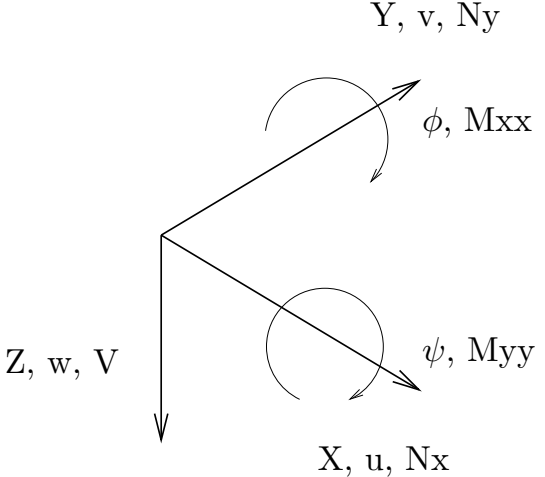

(b)

Figure 1. (a) Plate geometry (b) Associated boundary conditions. 


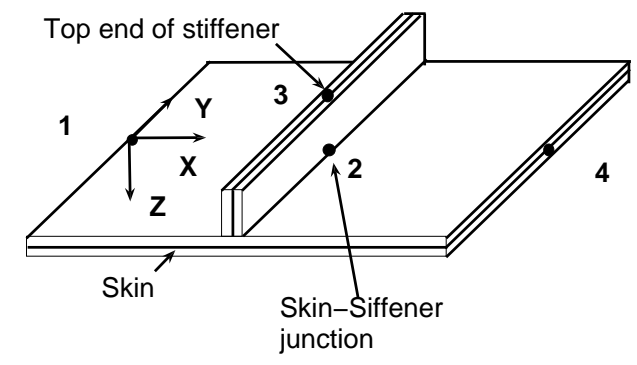

Figure 2. Schematic of a skin-stiffener structure

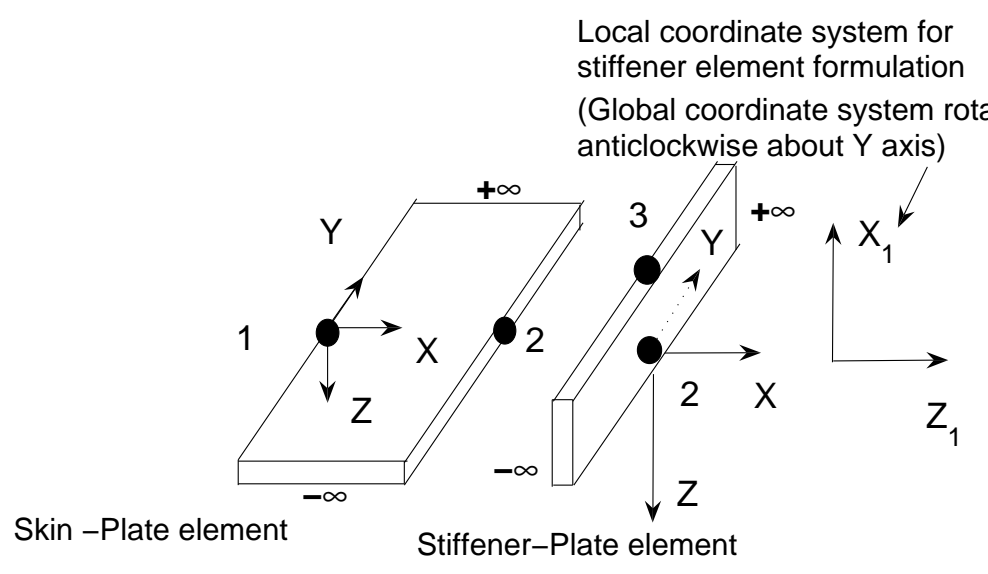

Figure 3. Plate-plate coupling. 


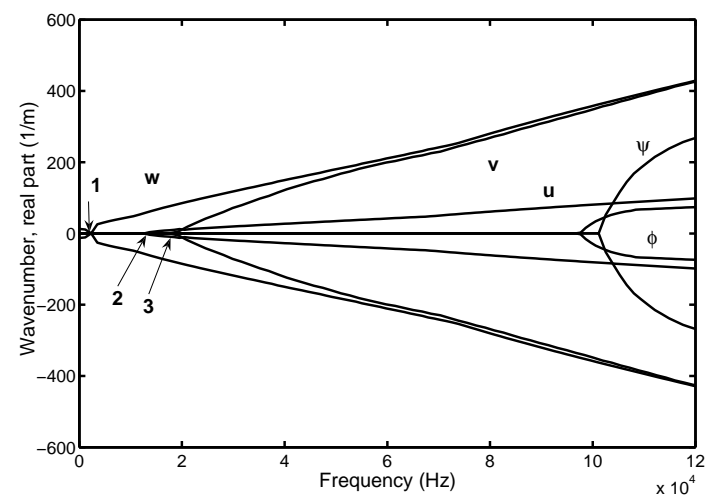

Figure 4. Real part of the wavenumbers.

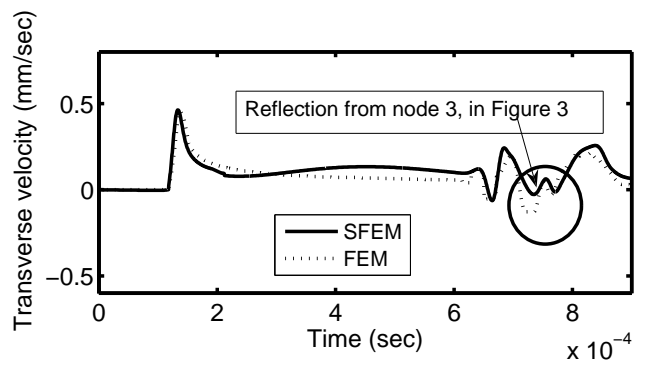

Figure 5. Transverse velocity response of skin-stiffener type structure using SFEM and FEM. 


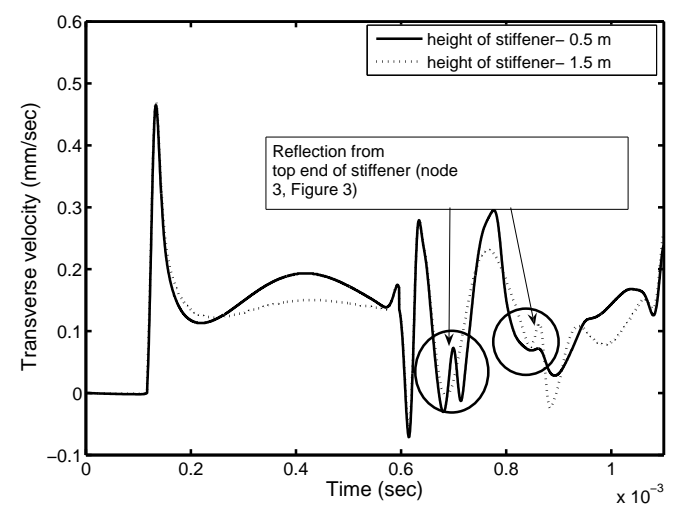

(a)

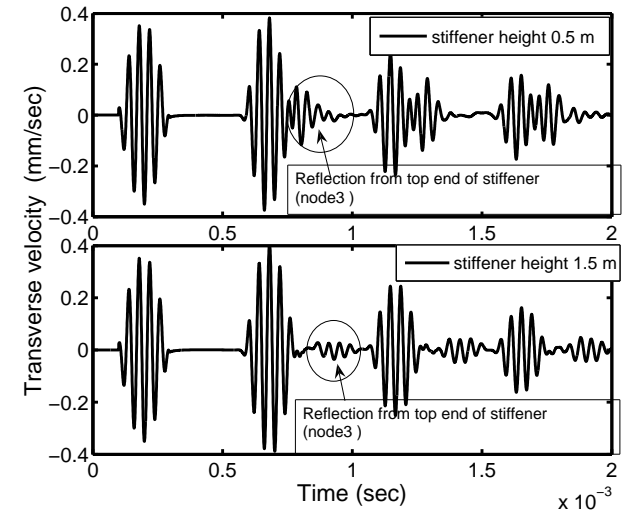

(b)

Figure 6. Transverse velocity response of skin-stiffener structure with different stiffener height (a) Applying broad band load.(b) Applying tone-burst load.

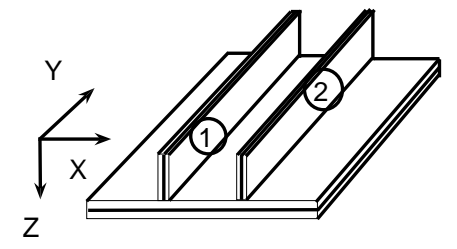

(a)

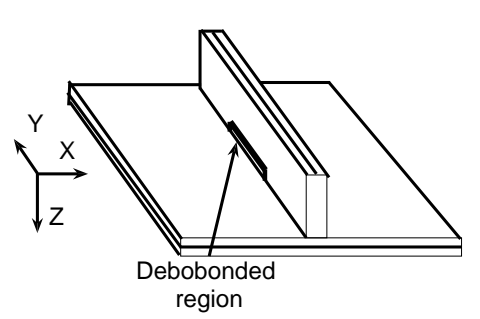

(b)

Figure 7. (a)Skin-stiffener structure with two stiffeners (b) Stiffened structure with a skin-stiffener debond. 


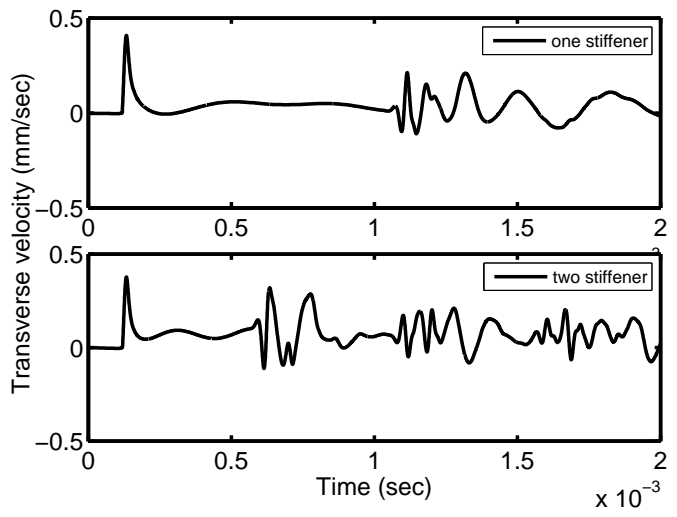

(a)

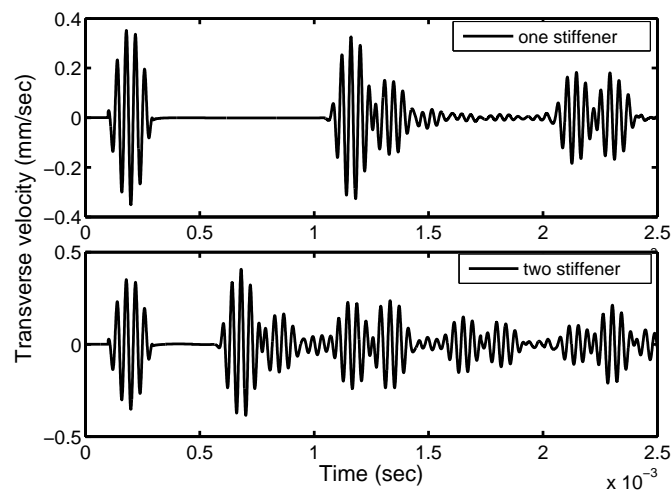

(b)

Figure 8. Transverse velocity response of skin-stiffener structure, varying the number of stiffeners, (a) Applying broad band load. (b) Applying tone-burst load.

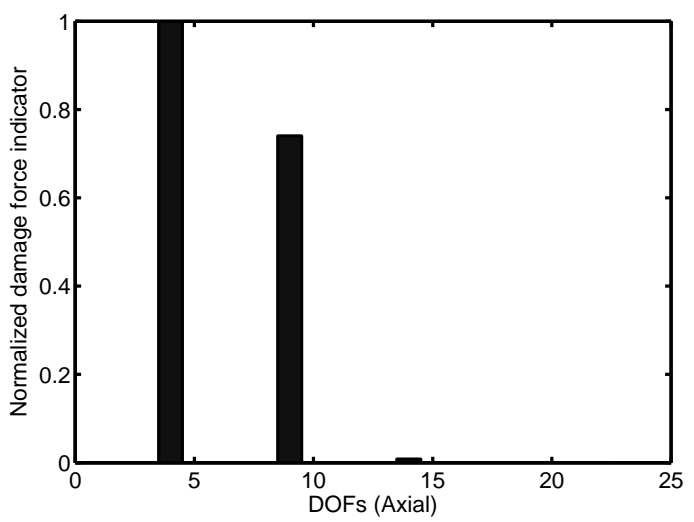

(a)

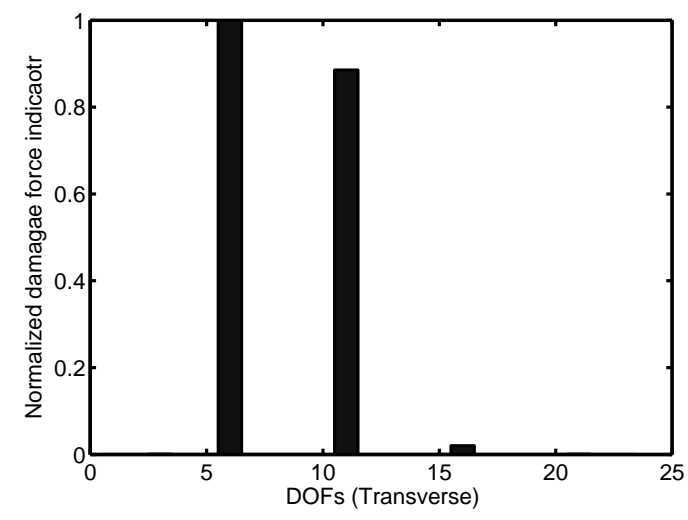

(b)

Figure 9. Normalized damage force indicators for a stiffened structure with skin-stiffener debond. 